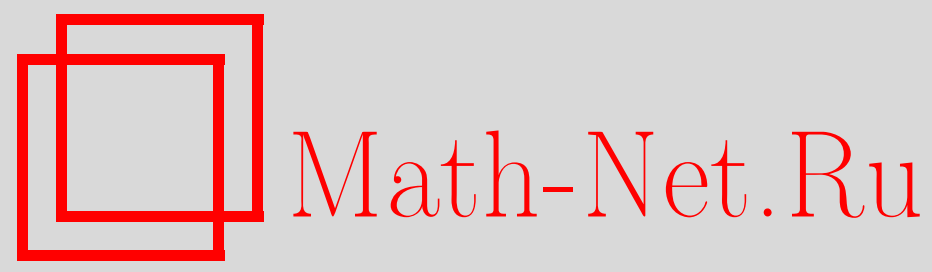

А. И. Кожанов, Об одном нелинейном нагруженном параболическом уравнении и о связанной с ним обратной задаче, Матем. заметки, 2004, том 76, выпуск 6, 840-853

DOI: https://doi.org/10.4213/mzm156

Использование Общероссийского математического портала Math-Net.Ru подразумевает, что вы прочитали и согласны с пользовательским соглашением http://www.mathnet.ru/rus/agreement

Параметры загрузки:

IP : 54.198 .64 .247

26 апреля 2023 г., $16: 34: 34$

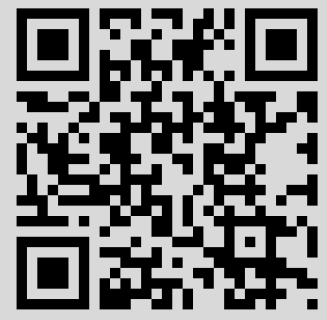




\section{ОБ ОДНОМ НЕЛИНЕЙНОМ НАГРУЖЕННОМ ПАРАБОЛИЧЕСКОМ УРАВНЕНИИ И О СВЯЗАННОЙ С НИМ ОБРАТНОЙ ЗАДАЧЕ}

\section{А. И. Кожанов}

В работе исследуется разрешимость нелокальной по времени краевой задачи для нелинейного параболического уравнения $u_{t}-\Delta u+c(\bar{u}(x, T)) u=f(x, t)$, где $\bar{u}(x, t)=$ $\alpha(t) u(x, t)+\int_{0}^{t} \beta(\tau) u(x, \tau) d \tau$ (здесь $\alpha(t)$ и $\beta(t)$ - заданные функции). Доказываются теоремы существования и единственности регулярных решений; показьвается, что полученные результаты могут быть использованы при исследовании разрешимости одной коэффиициентной обратной задачи.

Библиография: 11 названий.

Нагруженными уравнениями в литературе принято называть уравнения с частными производными, содержащие в коэффициентах значения тех или иных функционалов от решения [1]. Хорошо известными представителями подобных уравнений являются уравнения Кирхгофа и подобные им, исследованию которых посвяшено столь много работ, что даже краткое их перечисление займет очень много места. Различные классы нагруженных уравнений активно изучались в работах Дженалиева; итоги его исследований подведены в работе [2]. Как близкие к настоящей работе, отметим также статьи [3], [4], посвященные исследованию некоторых классов нагруженных уравнений параболического типа. И, наконец, заметим, что нагруженные уравнения естественным образом появляются при редукции некоторьх обратных задач к прямым задачам для уравнений составного типа - см. [3], [5], [6].

Перейдем к содержательной части работы.

Пусть $x$ есть точка ограниченной области $D$ пространства $\mathbb{R}^{n}$ с гладкой границей $\Gamma$, $t$ есть число из конечного интервала $(0, T), Q$ есть цилиндр $D \times(0, T)$. Далее, пусть $\alpha(t)$, $\beta(t), \varphi(x)$ и $\psi(x)$ - заданные при $t \in[0, T]$ и соответственно при $x \in \bar{D}$ функции, $c(\xi)-$ заданная при $\xi \in \mathbb{R}$ функция и $f(x, t)$ - заданная в $Q$ функция. Для заданной функции $h(t)$ определим величину $\bar{h}(t)$

$$
\bar{h}(t)=\alpha(t) h(t)+\int_{0}^{t} \beta(\tau) h(\tau) d \tau .
$$

Наконец, для функции $u(x, t)$, определенной в цилиндре $Q$, положим

$$
L u \equiv u_{t}-\Delta u+c(\bar{u}(x, T)) u,
$$

где $\Delta$ есть оператор Лапласа по переменным $x_{1}, \ldots, x_{n}$.

Работа выполнена при поддержке Российского фонда фундаментальных исследований, грант № 01-01-00796. 
КРАЕВАЯ ЗАДАЧА. Найти в $Q$ решение уравнения

$$
L u=f(x, t)
$$

удовлетворяющее условиям

$$
\begin{gathered}
u(x, 0)=\varphi(x) u(x, T)+\psi(x), \quad x \in D, \\
\left.u(x, t)\right|_{\Gamma \times(0, T)}=0 .
\end{gathered}
$$

Условие (2) данной краевой задачи представляет собой нелокальное условие, связывающее значения решения в различных точках границы [1]; тем самым, краевая задача $(1),(2)$ есть нелокальная краевая задача для нелинейного нагружсеного параболического уравнения. В указанной уже выше монографии [1], в работах [7], [8] приведены результаты о разрешимости некоторых нелокальных краевых задач для параболических уравнений, однако сразу же отметим, что все эти результаты соответствуют либо ситуации $\varphi(x) \equiv$ const, либо же дают существование обобщенного решения.

Теорема 1. Пусть имеют место включения $c(\xi) \in \operatorname{Lip}(\mathbb{R}), \alpha(t) \in L_{\infty}([0, T])$, $\beta(t) \in L_{1}([0, T]), \varphi(x) \in W_{\infty}^{1}(D), \psi(x) \in \stackrel{\circ}{W}_{2}^{1}(D) \cap L_{\infty}(D), f(x, t) \in L_{\infty}(Q)$, u nycmb выполняются условия

$$
0<c_{0} \leqslant c(\xi) \leqslant c_{1}<+\infty, \quad \xi \in \mathbb{R}, \quad \varphi_{0}=\|\varphi\|_{L_{\infty}(D)}<1
$$

Тогда краевая задача (1)-(3) имеет решение $u(x, t)$, принадлежсащее пространствy

$$
W_{2}^{2,1}(Q) \cap L_{\infty}\left(0, T ; \stackrel{\circ}{2}_{2}^{1}(D) \cap L_{\infty}(D)\right)
$$

ДокАЗАТЕЛЬСтво. Будем обозначать для краткости пространство $W_{2}^{2,1}(Q) \cap$ $L_{\infty}\left(0, T ; \mathfrak{W}_{2}^{1}(D) \cap L_{\infty}(D)\right)$ как пространство $V$; норму в $V$ будем определять естественньм образом:

$$
\|v\|_{V}=\|v\|_{W_{2}^{2,1}(Q)}+\|v\|_{L_{\infty}\left(0, T ; \stackrel{\circ}{2}_{2}^{1}(D) \cap L_{\infty}(D)\right)} \cdot
$$

Пусть $v(x, t)$ есть произвольная функция из этого пространства. Рассмотрим краевую задачу: найти в $Q$ решение уравнения

$$
L_{v} u \equiv u_{t}-\Delta u+c(\bar{v}(x, T)) u=f(x, t)
$$

удовлетворяющее условиям (2) и (3). Данная задача есть линейная нелокальная краевая задача; для доказательства разрешимости этой задачи в пространстве $V$ воспользуемся методом продолжения по параметру.

Пусть $\lambda$ есть число из отрезка [0,1]. Рассмотрим семейство краевых задач: найти в $Q$ решение уравнения $\left(1^{\prime}\right)$, удовлетворяющее условию

$$
u(x, 0)=\lambda \varphi(x) u(x, T)+\psi(x), \quad x \in D,
$$

и условию (3). 
Как обычно, обозначим через $\Lambda$ множество тех чисел $\lambda$ из отрезка $[0,1]$, для которых краевая задача $\left(1^{\prime}\right),\left(2_{\lambda}\right),(3)$ разрешима в пространстве $V$ для всех функций $f(x, t)$, $\varphi(x)$ и $\psi(x)$, удовлетворяющих условиям теоремы. Если это множество окажется непустым, одновременно открытьм и замкнутым, то оно, как известно, будет совпадать со всем отрезком $[0,1]$. Но тогда краевая задача $\left(1^{\prime}\right),\left(2_{1}\right),(3)$ будет иметь решение, принадлежащее пространству $V$. А это и будет означать разрешимость в пространстве $V$ задачи $\left(1^{\prime}\right),(2),(3)$.

Итак, нам необходимо доказать непустоту множества $\Lambda$, его открытость и замкнутость.

Чтобы доказать непустоту множества $\Lambda$, покажем, что число 0 есть элемент $\Lambda$.

Краевая задача $\left(1^{\prime}\right),\left(2_{0}\right),(3)$ есть обычная первая начально-краевая задача для линейного параболического уравнения. Хорошо известно [9], что при вьполнении условий теоремы эта задача имеет решение $u(x, t)$, принадлежащеепространству $W_{2}^{2,1}(Q) \cap$ $L_{\infty}\left(0, T ; W_{2}^{1}(D)\right)$. Вместе с тем при принадлежности функций $f(x, t)$ и $\psi(x)$ пространствам $L_{\infty}(Q)$ и $L_{\infty}(D)$ соответственно функция $u(x, t)$ будет ограниченной (существенно) и для нее почти всюду в $\bar{Q}$ будет иметь место оценка

$$
|u(x, t)| \leqslant \max \left\{\frac{1}{c_{0}}\|f\|_{L_{\infty}(Q)},\|\psi\|_{L_{\infty}(D)}\right\} .
$$

Этот факт доказан в [10] даже для обобщенного решения; тем более, он справедлив и для решения из пространства $W_{2}^{2,1}(Q) \cap L_{\infty}\left(0, T ; \stackrel{\circ}{W}_{2}^{1}(D)\right)$ (метод доказательства состоит в приближении функций $f(x, t), \psi(x)$ и $c(\bar{v}(x, T))$ гладкими, использовании для краевой задачи с гладкими данными классических результатов о существовании гладкого решения и о справедливости оценки принципа максимума [9] и в осуществлении предельного перехода).

Итак, краевая задача $\left(1^{\prime}\right),\left(2_{0}\right),(3)$ имеет решение, принадлежащее пространству $V$. Следовательно, множество $\Lambda$ непусто.

Открытость и замкнутость множества $\Lambda$, как правило, следуют из “хороших" априорных оценок. Докажем эти оценки.

Прежде всего заметим, что оценка (4) для решений $u(x, t)$ краевой задачи $\left(1^{\prime}\right),\left(2_{\lambda}\right)$, (3) дает неравенство

$$
\|u\|_{L_{\infty}\left(0, T ; L_{\infty}(D)\right)} \leqslant \max \left\{\frac{1}{c_{0}}\|f\|_{L_{\infty}(Q)}, \varphi_{0}\|u(x, T)\|_{L_{\infty}(D)}+\|\psi\|_{L_{\infty}(D)}\right\} .
$$

Используя элементарное неравенство $\max (\alpha, \beta+\gamma) \leqslant \max (\alpha, \beta)+\gamma$, справедливое для любых неотрицательных чисел $\alpha, \beta, \gamma$, мы можем далее получить неравенство

$$
\|u\|_{L_{\infty}\left(0, T ; L_{\infty}(D)\right)} \leqslant \max \left\{\frac{1}{c_{0}}\|f\|_{L_{\infty}(Q)},\|\psi\|_{L_{\infty}(D)}\right\}+\varphi_{0}\|u\|_{L_{\infty}\left(0, T ; L_{\infty}(D)\right)}
$$

Условие $\varphi_{0}<1$ позволяет нам получить первую равномерную по $\lambda$ оценку решений краевой задачи $\left(1^{\prime}\right),\left(2_{\lambda}\right),(3)$ :

$$
\|u\|_{L_{\infty}\left(0, T ; L_{\infty}(D)\right)} \leqslant \frac{1}{1-\varphi_{0}} \max \left\{\frac{1}{c_{0}}\|f\|_{L_{\infty}(Q)},\|\psi\|_{L_{\infty}(D)}\right\} .
$$


Элементарный анализ равенства

$$
\int_{0}^{T} \int_{D} L_{v} u \cdot u d x d t=\int_{0}^{T} \int_{D} f u d x d t
$$

и вновь условие $\varphi_{0}<1$ дают вторую равномерную по $\lambda$ оценку

$$
\int_{D} u^{2}(x, T) d x+\int_{0}^{T} \int_{D}\left[u^{2}+\sum_{i=1}^{n} u_{x_{i}}^{2}\right] d x d t \leqslant M_{1}\left[\int_{0}^{T} \int_{D} f^{2} d x d t+\int_{D} \psi^{2} d x\right]
$$

где $M_{1}$ есть число, определяемое лишь числами $c_{0}$ и $\varphi_{0}$.

Проанализируем следующее равенство:

$$
\int_{0}^{T} \int_{D} L_{v} u \cdot\left(u_{t}-\Delta u\right) d x d t=\int_{0}^{T} \int_{D} f\left(u_{t}-\Delta u\right) d x d t
$$

Интегрируя по частям, опять используя условие $\varphi_{0}<1$, а также используя оценку (6), второе основное неравенство для эллиптических операторов [9] и неравенство Юнга, получаем, что для функции $u(x, t)$ будет справедлива оценка

$$
\begin{aligned}
& \int_{0}^{T} \int_{D}\left[u_{t}^{2}+\sum_{i, j=1}^{n} u_{x_{u} x_{j}}^{2}\right] d x d t+\sum_{i=1}^{n} \int_{D} u_{x_{i}}^{2}(x, T) d x \\
& \leqslant M_{2}\left[\int_{0}^{T} \int_{D} f^{2} d x d t+\int_{D} \psi^{2} d x+\sum_{i=1}^{n} \int_{D} \psi_{x_{i}}^{2} d x\right]
\end{aligned}
$$

где постоянная $M_{2}$, зависит от чисел $c_{1}$ и $M_{1}$, от функции $\varphi(x)$ и от области $D$.

Из оценок (6) и (7) и из условий на функции $\varphi(x)$ и $\psi(x)$ вытекает оценка

$$
\sum_{i=1}^{n} \int_{D} u_{x_{i}}^{2}(x, 0) d x \leqslant M_{3}\left[\int_{0}^{T} \int_{D} f^{2} d x d t+\int_{D} \psi^{2} d x+\sum_{i=1}^{n} \int_{D} \psi_{x_{i}}^{2} d x\right] .
$$

Интегрируя по частям в равенстве

$$
\int_{0}^{t} \int_{D} L_{v} u\left(u_{\tau}-\Delta u\right) d x d \tau=\int_{0}^{t} \int_{D} f\left(u_{\tau}-\Delta u\right) d x d \tau
$$

где $t$ есть произвольное число из отрезка $[0, T]$, нетрудно получить оценку

$$
\sum_{i=1}^{n} \int_{D} u_{x_{i}}^{2}(x, t) d x \leqslant M_{4}\left[\int_{0}^{T} \int_{D} f^{2} d x d t+\int_{D} \psi^{2} d x+\sum_{i=1}^{n} \int_{D} \psi_{x_{i}}^{2} d x\right]
$$

с постоянной $M_{4}$, зависящей, как и предыдущие постоянные $M_{1}, M_{2}, M_{3}$, лишь от чисел $c_{0}$ и $c_{1}$, функции $\varphi(x)$ и от области $D$. Вместе с оценками $(5)-(7)$ последняя оценка дает равномерную по $\lambda$ оценку $u(x, t)$ решений краевой задачи $\left(1^{\prime}\right),\left(2_{\lambda}\right),(3)$ в пространстве $V$ :

$$
\|u\|_{V} \leqslant \frac{1}{1-\varphi_{0}} \max \left\{\frac{1}{c_{0}}\|f\|_{L_{\infty}(Q)}, \| \psi_{L_{\infty}(D)}\right\}+M_{0}\left[\|f\|_{L_{2}(Q)}+\|\psi\|_{W_{2}^{1}(D)}\right] .
$$


Эта оценка и позволит нам доказать открытость и замкнутость множества $\Lambda$.

Пусть $\left\{\lambda_{m}\right\}$ есть последовательность элементов множества $\Lambda$, сходящаяся к числу $\lambda_{0}$. Покажем, что $\lambda_{0}$ также будет элементом $\Lambda$.

Всякому числу $\lambda_{m}$ соответствует функция $u_{m}(x, t)$, принадлежащая пространству $V$ и являющаяся решением краевой задачи $\left(1^{\prime}\right),\left(2_{\lambda_{m}}\right),(3)$. Для семейства функций $\left\{u_{m}(x, t)\right\}$ будет справедлива равномерная по $m$ оценка $(8)$. Из этой оценки вытекает, что найдутся функция $u(x, t)$ из пространства $W_{2}^{2,1}(Q)$ и подпоследовательность $\left\{u_{m_{k}}(x, t)\right\}$ последовательности $\left\{u_{m}(x, t)\right\}$ такие, что при $k \rightarrow \infty$ имеют место сходимости $u_{m_{k}}(x, t) \rightarrow u(x, t)$ слабо в $W_{2}^{2,1}(Q)$ и почти всюду в $\bar{Q}$. Из этих сходимостей следует, что предельная функция $u(x, t)$ будет решением уравнения $\left(1^{\prime}\right)$ и что для нее будут выполняться условия $\left(2_{\lambda_{0}}\right)$ и $(3)$. Оценка (8) для решений задачи $\left(1^{\prime}\right),\left(2_{\lambda_{0}}\right),(3)$ далее дает, что функция $u(x, t)$ будет принадлежать пространству $V$. Отсюда и вытекает, что число $\lambda_{0}$ будет элементом множества $\Lambda$.

Принадлежность (произвольной) предельной для множества $\Lambda$ точки ему же и означает его замкнутость.

Докажем теперь открытость множества $\Lambda$.

Пусть число $\lambda_{0}$ есть элемент множества $\Lambda$. Множество $\Lambda$ будет открытым, если числа $\lambda=\lambda_{0}+\hat{\lambda}$ при малой величине $|\hat{\lambda}|$ также будут принадлежать ему же. Пусть $w(x, t)$ есть произвольная функция из пространства $V$. Рассмотрим краевую задачу: найти в $Q$ решение уравнения $\left(1^{\prime}\right)$, удовлетворяющее условию

$$
u(x, 0)=\lambda_{0} \varphi(x) u(x, T)+\hat{\lambda} \varphi(x) w(x, T)+\psi(x), \quad x \in D
$$

и условию (3). Согласно определению множества $\Lambda$ эта задача имеет решение $u(x, t)$, принадлежащее пространству $V$. Тем самьп, краевая задача $\left(1^{\prime}\right),\left(2_{\lambda_{0}}^{\prime}\right),(3)$ порождает оператор $G$, переводящий пространство $V$ в себя: $G(w)=u$. Покажем, что при малой величине $|\hat{\lambda}|$ этот оператор будет сжимающим.

Пусть $w_{1}(x, t)$ и $w_{2}(x, t)$ - функции из пространства $V, u_{1}(x, t)$ и $u_{2}(x, t)$ - соответствующие им образы при действии оператора $G$. Обозначим $w(x, t)=w_{1}(x, t)-w_{2}(x, t)$, $u(x, t)=u_{1}(x, t)-u_{2}(x, t)$. Для функции $u(x, t)$ выполняется уравнение

$$
u_{t}-\Delta u+c(\bar{v}(x, T)) u=0,
$$

условие

$$
u(x, 0)=\lambda_{0} \varphi(x) u(x, T)+\hat{\lambda} \varphi(x) w(x, T), \quad x \in D,
$$

а также условие (3). Оценка (8) дает для функции $u(x, T)$ неравенство

$$
\|u\|_{V} \leqslant \frac{|\hat{\lambda}| \varphi_{0}}{1-\varphi_{0}}\|w(x, T)\|_{L_{\infty}(D)}+M_{0}^{\prime}|\hat{\lambda}| \int_{D}\left[w^{2}(x, T)+\sum_{i=1}^{n} w_{x_{i}}^{2}(x, T)\right] d x .
$$

Из этого неравенства очевидньм образом вытекает неравенство

$$
\|u\|_{V} \leqslant M \cdot|\hat{\lambda}|\|w\|_{V}
$$

Если теперь число $\hat{\lambda}$ будет таким, что будет выполняться неравенство $M|\hat{\lambda}|<1$, то оператор $G$ как раз и станет сжимающим оператором. У сжимающегося оператора есть 
неподвижная точка - функция $u(x, t)$ такая, что $G(u)=u$. Для этой функции будет выполняться уравнение $\left(1^{\prime}\right)$, условие $(3)$ и условие

$$
u(x, 0)=\left(\lambda_{0}+\hat{\lambda}\right) \varphi(x) u(x, T)+\psi(x), \quad x \in D .
$$

Другими словами, функция $u(x, t)$ будет решением краевой $\left(1^{\prime}\right),\left(2_{\lambda}\right),(3)$ и будет принадлежать пространству $V$. А это и означает, что число $\lambda_{0}+\hat{\lambda}$ есть элемент множества $\Lambda$, и далее - что множество $\Lambda$ открыто.

Как уже говорилось вьше, из доказанного следует, что краевая задача $\left(1^{\prime}\right),(2),(3)$ при принадлежности функции $v(x, t)$ пространству $V$ будет иметь решение $u(x, t)$, принадлежащее снова пространству $V$. Но тогда эта краевая задача порождает оператор $\Phi$, переводящий пространство $V$ в себя: $\Phi(v)=u$. Покажем, что оператор $\Phi$ имеет в $V$ неподвижные точки.

Для решений краевой задачи $\left(1^{\prime}\right),(2),(3)$ вьполняется оценка (8). Следовательно, оператор $\Phi$ любой шар пространства $V$ радиуса $R$ такого, что

$$
R \geqslant \frac{1}{1-\varphi_{0}} \max \left\{\frac{1}{c_{0}}\|f\|_{L_{\infty}(Q)},\|\psi\|_{L_{\infty}(D)}\right\}+M_{0}\left[\|f\|_{L_{2}(Q)}+\|\psi\|_{W_{2}^{1}(D)}\right],
$$

будет переводить в себя.

Покажем теперь, что оператор $\Phi$ будет вполне непрерьвен.

Пусть последовательность функций $\left\{v_{m}(x, t)\right\}$ есть ограниченная последовательность из пространства $V,\left\{u_{m}(x, t)\right\}$ есть последовательность образов функций $v_{m}(x, t)$ при действии оператора $\Phi$. Вновь из оценок (5)-(7) вытекает, что последовательность $\left\{u_{m}(x, t)\right\}$ будет ограничена в пространстве $V$. Далее, заметим, что последовательность $\left\{\bar{v}_{m}(x, T)\right\}$ будет ограниченной последовательностью в пространстве $W_{2}^{1}(D)$. Из ограниченности последовательностей $\left\{v_{m}(x, t)\right\},\left\{u_{m}(x, t)\right\}$ и $\left\{\bar{v}_{m}(x, T)\right\}$ следует, что существуют подпоследовательности $\left\{v_{m_{k}}(x, t)\right\},\left\{u_{m_{k}}(x, t)\right\}$ и $\left\{\bar{v}_{m_{k}}(x, t)\right\}$ соответствующих последовательностей, и функции $v(x, t), u(x, t)$ и $\{\bar{v}(x, t)\}$ такие, что при $k \rightarrow \infty$ имеют место сходимости

- $v_{m_{k}}(x, t) \rightarrow v(x, t)$ слабо в $W_{2}^{2,1}(Q)$ и почти всюду в $\bar{Q}$,

- $u_{m_{k}}(x, t) \rightarrow u(x, t)$ слабо в $W^{2,1}(Q)$ и почти всюду в $\bar{Q}$,

- $\bar{v}_{m_{k}}(x, T) \rightarrow \bar{v}(x, T)$ почти всюду в $\bar{D}$.

Из этих сходимостей следует, что функции $v(x, t)$ и $u(x, t)$ будут связаны уравнением $\left(1^{\prime}\right)$; очевидно при этом, что функция $u(x, t)$ будет ограничена (существенно) и что для нее будет вьполняться условие $(2)$. Обозначим $w_{k}(x, t)=u_{m_{k}}(x, t)-u(x, t)$. Имеет место равенство

$$
w_{k_{t}}-\Delta w_{k}+c\left(\bar{v}_{m_{k}}(x, T)\right) w_{k}=\left[c(\bar{v}(x, T))-c\left(\bar{v}_{m_{k}}(x, T)\right)\right] u .
$$

Это равенство, оценка (8) для функции $w_{k}$, ограниченность функции $u(x, t)$, липшицевость функции $c(\xi)$ и сходимость последовательности $\left\{\bar{v}_{m_{k}}(x, T)-\bar{v}(x, T)\right\}$ почти всюду в $\bar{D}$ к нулевой функции дают нам сходимость

$$
\left\|w_{k}\right\|_{V} \rightarrow 0 \quad \text { при } k \rightarrow \infty .
$$

Другими словами, для всякой ограниченной в пространстве $V$ последовательности $\left\{v_{m}(x, t)\right\}$ из последовательности $\left\{\Phi v_{m}\right\}$ можно извлечь сильно сходящуюся 
подпоследовательность. А это и означает, что оператор $\Phi$ вполне непрерьвен в пространстве $V$.

Вполне непрерьвность оператора $\Phi$, его свойство переводить шар пространства $V$ достаточно большого радиуса в себя и теорема Шаудера и дают нам, что оператор $\Phi$ имеет в пространстве $V$ неподвижную точку. Эта неподвижная точка - т.е. функция $u(x, t)$ из пространства $V$ - и будет искомым решением краевой задачи $(1)-(3)$.

Теорема доказана.

Обсудим вопрос о единственности решений краевой задачи (1)-(3).

Введем обозначения

$$
K_{0}=\frac{1}{1-\varphi_{0}} \max \left\{\frac{1}{c_{0}}\|f\|_{L_{\infty}(Q)},\|\psi\|_{L_{\infty}(D)}\right\}, \quad a_{0}=|\alpha(T)|+\int_{0}^{T}|\beta(t)| d t .
$$

ТЕОРема 2. Пусть выполняются все условия теоремы 1, а также условие

$$
C K_{0} a_{0}<1-\varphi_{0},
$$

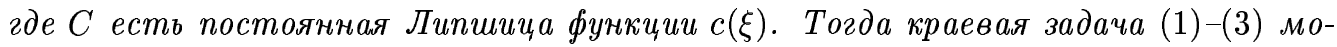
жет иметь в пространстве $V$ лишь одно решение.

ДокАЗАТЕЛЬСтво. Предположим, что краевая задача (1)-(3) имеет в пространстве $V$ два решения $u(x, t)$ и $v(x, t)$. Обозначим $w(x, t)=u(x, t)-v(x, t)$. Для функции $w(x, t)$ в $Q$ вьполняется уравнение

$$
w_{t}-\Delta w+c(\bar{u}(x, T)) w=[c(\bar{v}(x, T))-c(\bar{u}(x, T))] v,
$$

условие

$$
w(x, 0)=\varphi(x) w(x, T),
$$

а также условие (3). Оценка (5) для функции $w(x, t)$ вместе с липшицевостью функции $c(\xi)$ дает оценку

$$
\|w\|_{L_{\infty}\left(0, T ; L_{\infty}(D)\right)} \leqslant \frac{1}{1-\varphi_{0}} \cdot C \cdot\|\bar{w}(x, T)\|_{L_{\infty}(D)}\|v\|_{L_{\infty}\left(0, T ; L_{\infty}(D)\right)} .
$$

Поскольку функция $v(x, t)$ есть решение краевой задачи $(1)-(3)$, то для нее будет вьполняться оценка

$$
\|v\|_{L_{\infty}\left(0, T ; L_{\infty}(D)\right)} \leqslant K_{0}
$$

Далее, для функции $\bar{w}(x, T)$ выполняется неравенство

$$
\|\bar{w}(x, T)\|_{L_{\infty}(D)} \leqslant a_{0}\|w\|_{L_{\infty}\left(0, T ; L_{\infty}(D)\right)} .
$$

Последние два неравенства дают возможность продолжить оценку (9):

$$
\|w\|_{L_{\infty}\left(0, T ; L_{\infty}(D)\right)} \leqslant \frac{C K_{0} a_{0}}{1-\varphi_{0}}\|w\|_{L_{\infty}\left(0, T ; L_{\infty}(D)\right)} .
$$

Очевидно, что вследствие условия $C K_{0} a_{0}<1-\varphi_{0}$ данное неравенство возможно лишь в случае

$$
\|w\|_{L_{\infty}\left(0, T ; L_{\infty}(D)\right)}=0 .
$$

А это и дает совпадение функций $u(x, t)$ и $v(x, t)$ почти всюду в $Q$.

Теорема доказана.

Приведем еше один вариант теоремы разрешимости краевой задачи (1)-(3). 
ТЕОРема 3. Пусть для функиии с $(\xi)$ существуют положсительные числа $c_{0}, c_{1}$ и то такие, что выполняются неравенства

$$
c_{0} \leqslant c(\xi) \leqslant c_{1} \quad n p u \quad \xi \in\left[-m_{0}, m_{0}\right] .
$$

Кроме того, пусть имеют место включения

$$
\begin{array}{ll}
c(\xi) \in \operatorname{Lip}\left(\left[-m_{0}, m_{0}\right]\right), \quad \alpha(t) \in L_{\infty}([0, T]), & \beta(t) \in L_{1}([0, T]), \\
\varphi(x) \in W_{\infty}^{1}(D), \quad \psi(x) \in \stackrel{\circ}{W}_{2}^{1}(D) \cap L_{\infty}(D), & f(x, t) \in L_{\infty}(Q),
\end{array}
$$

и пусть выполняются условия

$$
\varphi_{0}<1, \quad a_{0} K_{0} \leqslant m_{0} .
$$

Тогда краевая задача (1)-(3) имеет решение, принадлежащее пространству $V$.

ДоказАТЕЛЬСТво. Определим функцию $\hat{c}(\xi)$ :

$$
\hat{c}(\xi)= \begin{cases}c\left(m_{0}\right), & \text { если } \xi>m_{0}, \\ c(\xi), & \text { если } \xi \in\left[-m_{0}, m_{0}\right], \\ c\left(-m_{0}\right), & \text { если } \xi<-m_{0} .\end{cases}
$$

Очевидно, что функция $\hat{c}(\xi)$ удовлетворяет условию Липшица на всей числовой оси.

Рассмотрим краевую задачу: найти функцию $u(x, t)$, являющуюся в $Q$ решением уравнения

$$
u_{t}-\Delta u+\hat{c}(\bar{u}(x, T)) u=f(x, T)
$$

и удовлетворяющую условиям (2) и (3).

Согласно теореме 1 краевая задача $(10),(2),(3)$ имеет решение $u(x, t)$, принадлежашее пространству $V$. Для этого решения будет выполняться оценка

$$
|u(x, t)| \leqslant K_{0}
$$

для почти всех точек $(x, t)$ множества $\bar{Q}$. Следовательно, для почти всех точек $x$ из множества $\bar{D}$ будет выполняться оценка

$$
|\bar{u}(x, T)| \leqslant a_{0} K_{0} .
$$

Условие теоремы влечет за собой равенство $\hat{c}(\bar{u}(x, T))=c(\bar{u}(x, T))$. Другими словами, найденная функция $u(x, t)$ - т.е. решение краевой задачи $(10),(2),(3)$ - будет и решением краевой задачи (1)-(3).

Теорема доказана.

ЗАМЕЧАнИЕ 1. Изначально функция $c(\cdot)$ может быть функцией не только числа $\xi$, но и точки $(x, t)$ множества $\bar{Q}$. Необходимо лишь потребовать, чтобы условия теорем 1 и 3 , накладываемые на функцию $c(x, t, \xi)$, выполнялись бы равномерно на множестве $\bar{Q}$.

Покажем, что полученные выше результаты могут быть использованы при исследовании некоторых обратньгх задач.

Пусть $f(x, t),(x, t) \in \bar{Q}, \alpha(t), t \in[0, T], u_{0}(x)$ и $u_{1}(x), x \in \bar{D},-$ заданные функции. 
ОБРАТНАЯ ЗАДАЧА. Найти функции $u(x, t)$ и $q(x)$, связанные в $Q$ уравнением

$$
u_{t}-\Delta u+q(x) u=f(x, t)
$$

при выполнении условий

$$
\begin{gathered}
\left.u(x, t)\right|_{\Gamma \times(0, T)}=\mu(x, t), \\
u(x, 0)=u_{0}(x), \quad x \in D \\
\int_{0}^{T} \alpha(t) u(x, t) d t=u_{1}(x), \quad x \in D .
\end{gathered}
$$

В обратной задаче (11)-(14) условия (12) и (13) являются условиями прямой задачи, условие же (14) есть интегральное условие переопределения, необходимое для нахождения вместе с решением и неизвестного коэффициента. Разрешимость обратной задачи для параболического уравнения с неизвестным коэффициентом при решении и с интегральным условием переопределения в пространствах соболевского типа изучалась ранее в [11]; среди условий, гарантирующих существование решений, в указанной работе фигурировали условия неотрицательности функций $f(x, t), f_{t}(x, t), \mu(x, t)$ и $\mu_{t}(x, t)$. В нашей же работе разрешимость обратной задачи (11)-(14) будет доказана при выполнении иных, нежели указанные условия неотрицательности, условий.

Пусть вьполняются условия

$$
\begin{gathered}
f(x, t) \in L_{\infty}(Q), \quad u_{0}(x) \in W_{2}^{1}(D) \cap L_{\infty}(D), \\
\mu(x, t) \in L_{\infty}(\Gamma \times[0, T]), \\
\exists \mathscr{M}(x, t) \text { такая, что } \mathscr{M}(x, t) \in V,\left.\mathscr{M}(x, t)\right|_{\Gamma \times(0, T)}=\mu(x, t), \\
\mu(x, 0)=u_{0}(x) \text { при } x \in \Gamma ; \\
0<k_{0} \leqslant u_{1}(x) \leqslant k_{1}<+\infty \text { почти всюду в } \bar{D} ; \\
\alpha(t) \in C^{1}([0, T]) ; \\
u_{1}(x) \in W_{2}^{2}(D) \cap L_{\infty}(D), \quad \\
a(x) \equiv \int_{0}^{T} \alpha(t) f(x, t) d t+\Delta u_{1}(x)+\alpha(0) u_{0}(x) \geqslant m_{0}>0 \text { почти всюду в } \bar{D} .
\end{gathered}
$$

Определим функцию $v_{0}(x, t)$ как решение краевой задачи

$$
\begin{gathered}
v_{0_{t}}-\Delta v_{0}+\frac{a(x)}{u_{1}(x)} v_{0}=f(x, t), \quad(x, t) \in Q, \\
\left.v_{0}\right|_{\Gamma \times(0, T)}=\mu(x, t), \\
v_{0}(x, 0)=u_{0}(x), \quad x \in D .
\end{gathered}
$$

Как уже говорилось вьше (при исследовании разрешимости краевой задачи $\left(1^{\prime}\right),(2)$, $(3))$, при вьполнении условий $(15),(16)$ и $(18)$ функция $v_{0}(x, t)$ будет принадлежать пространству $V$ и для нее почти всюду в множестве $\bar{Q}$ будет выполняться оценка

$$
\left|v_{0}(x, t)\right| \leqslant \max \left\{\frac{k_{1}}{m_{0}}\|f\|_{L_{\infty}(Q)},\left\|u_{0}\right\|_{L_{\infty}(D)},\|\mu\|_{L_{\infty}(\Gamma \times[0, T])}\right\}=N_{0} .
$$


Определим величину $\bar{h}(T)$ :

$$
\bar{h}(T)=-\alpha(T) h(T)+\int_{0}^{T} \alpha^{\prime}(t) h(t) d t .
$$

Далее, пусть $m_{1}$ есть произвольное число из интервала $\left(0, m_{0}\right)$. Определим числа $a_{0}$, $N_{1}$ и $N_{2}$ :

$$
a_{0}=|\alpha(T)|+\int_{0}^{T}\left|\alpha^{\prime}(t)\right| d t, \quad N_{1}=\frac{a_{0} k_{1} N_{0}}{k_{0}\left(m_{0}-m_{1}\right)}, \quad N_{2}=N_{1} N_{0} .
$$

ТЕОРЕма 4. Пусть выполняются условия (15)-(18), а также условия

$$
\begin{gathered}
N_{1}<1, \quad a_{0}\left(\frac{N_{2}}{1-N_{1}}+N_{0}\right) \leqslant m_{1}, \\
\int_{0}^{T} h(t) \mu(x, t) d t=u_{1}(x) \quad \text { npu } \quad x \in \Gamma .
\end{gathered}
$$

Тогда обратная задача (11)-(14) имеет решение $\{u(x, t), q(x)\}$ такое, что $u(x, t)$ $\in V, q(x) \in L_{\infty}(D), q(x)>0$ почти всюду на множестве $\bar{D}$.

ДокАЗАТЕЛЬСТво. Определим функцию

$$
c_{0}(\xi)= \begin{cases}\xi, & \text { если }|\xi| \leqslant m_{1}, \\ m_{1}, & \text { если } \xi>m_{1}, \\ -m_{1}, & \text { если } \xi<-m_{1} .\end{cases}
$$

Рассмотрим краевую задачу: найти в $Q$ решение уравнения

$$
w_{t}-\Delta w+\frac{a(x)+c_{0}\left(\bar{w}(x, T)+\bar{v}_{0}(x, T)\right)}{u_{1}(x)} w=-\frac{1}{u_{1}(x)}\left[\bar{w}(x, T)+\bar{v}_{0}(x, T)\right] v_{0},
$$

удовлетворяющее условиям

$$
\begin{gathered}
\left.w(x, t)\right|_{\Gamma \times(0, T)}=0, \\
w(x, 0)=0, \quad x \in D .
\end{gathered}
$$

Исследование разрешимости данной краевой задачи проведем с помощю метода неподвижной точки.

Пусть $v(x, t)$ есть произвольная функция из пространства $V$. Рассмотрим краевую задачу: найти в $Q$ решение уравнения

$$
w_{t}-\Delta w+\frac{a(x)+c_{0}\left(\bar{w}(x, T)+\bar{v}_{0}(x, T)\right)}{u_{1}(x)} w=-\frac{1}{u_{1}(x)}\left[\bar{v}(x, T)+\bar{v}_{0}(x, T)\right] v_{0},
$$

удовлетворяющее условиям (22) и (23).

Из теоремы 1 и замечания 1 следует, что краевая задача $\left(21^{\prime}\right),(22),(23)$ имеет решение $w(x, t)$, принадлежащее пространству $V$. Тем самым, эта краевая задача порождает оператор $\Phi$, переводящий пространство $V$ в себя. Покажем, что этот оператор будет 
переводить некоторое замкнутое выпуклое ограниченное множество пространства $V$ в себя.

Определим требуемое множество так:

$$
W_{R_{1} R_{2}}=\left\{v \in V:\|v\|_{L_{\infty}(Q)} \leqslant R_{1},\|v\|_{W_{2}^{2,1}(Q) \cap L_{\infty}\left(0, T ; W_{2}^{1}(D)\right)} \leqslant R_{2}\right\} .
$$

Покажем, что числа $R_{1}$ и $R_{2}$ можно выбрать так, что множество $W_{R_{1}} R_{2}$ и будет искомым.

Для решений краевой задачи $\left(21^{\prime}\right),(22),(23)$ почти всюду на множестве $\bar{Q}$ выполняется оценка

$$
|w(x, t)| \leqslant \frac{a_{0} k_{1} N_{0}}{k_{0}\left(m_{0}-m_{1}\right)}\|v\|_{L_{\infty}(Q)}+\frac{a_{0} k_{1} N_{0}^{2}}{k_{0}\left(m_{0}-m_{1}\right)}=N_{1}\|v\|_{L_{\infty}(Q)}+N_{2} .
$$

Выберем число $R_{1}$ так, чтобы вьполнялось неравенство $\left(1-N_{1}\right) R_{1} \geqslant N_{2}$ (вследствие условия $N_{1}<1$ это возможно). Тогда из оценки (24) будет вытекать оценка

$$
\|w\|_{L_{\infty}(Q)} \leqslant R_{1}
$$

Повторяя далее доказательство оценки (8) и используя оценку (25), нетрудно установить, что для решений краевой задачи (21)-(23) будет выполняться оценка

$$
\|w\|_{W_{2}^{2,1}(Q) \cap L_{\infty}\left(0, T ; \stackrel{\circ}{2}_{2}^{1}(D)\right)} \leqslant N
$$

где постоянная $N$ зависит лишш от исходных данных задачи и от числа $R_{1}$. Выберем число $R_{2}$ большим данного числа $N$. Вместе с выбранньгм ранее (и зафиксированным) числом $R_{1}$ такой выбор числа $R_{2}$ и даст нам, что оператор $\Phi$ будет переводить множество $W_{R_{1} R_{2}}$ в себя.

Вполне непрерывность оператора $\Phi$ доказьвается полностью аналогично доказательству вполне непрерьвности соответствующего оператора, возникающего при доказательстве второй части теоремы 1 (при этом существенно используется вытекающее из неравенств (24) и (26) свойство оператора $\Phi$ переводить ограниченные множества пространства $V$ в ограниченные).

Доказанные вьше свойства оператора $\Phi$ и теорема Шаудера дают нам существование неподвижной точки у этого оператора - т.е. существование функции $w(x, t)$, принадлежащей пространству $V$ и являющейся решением краевой задачи (21)-(23).

Из неравенства (24) вытекает, что для решений краевой задачи (21)-(23) будет выполняться оценка

$$
\|w\|_{L_{\infty}(Q)} \leqslant \frac{N_{2}}{1-N_{1}}
$$

Из этой оценки, в свою очередь, вытекает оценка

$$
\left|\bar{w}(x, T)+\bar{v}_{0}(x, T)\right| \leqslant a_{0}\left(\frac{N_{2}}{1-N_{1}}+N_{0}\right) .
$$

Условие (19) и эта оценка дают равенство

$$
c_{0}\left(\bar{w}(x, T)+\bar{v}_{0}(x, T)\right)=\bar{w}(x, T)+\bar{v}_{0}(x, T)
$$


Другими словами, найденное решение краевой задачи (21)-(23) будет решением уравнения

$$
w_{t}-\Delta w+\frac{a(x)+\bar{w}(x, T)+\bar{v}_{0}(x, T)}{u_{1}(x)} w=-\left[\bar{w}(x, T)+\bar{v}_{0}(x, T)\right] v_{0} .
$$

Определим функцию $u(x, t)$ равенством $u(x, t)=w(x, t)+v_{0}(x, t)$. Очевидно, что для этой функции вьполняются условия (12) и (13). Далее, будет выполняться равенство

$$
u_{t}-\Delta u+\frac{a(x)+\bar{u}(x, T)}{u_{1}(x)} u=f
$$

Это равенство означает, что для функции $u(x, t)$ и функции $q(x)$, определенной равенством

$$
q(x)=\frac{a(x)+\bar{u}(x, T)}{u_{1}(x)},
$$

уравнение (28) превратится в уравнение (11).

Умножим полученное уравнение $(11)$ на функцию $\alpha(t)$ и проинтегрируем полученное равенство по отрезку $[0, T]$. Обозначив для краткости через $\psi(x)$ функцию

$$
\psi(x)=\int_{0}^{T} \alpha(t) u(x, t) d t
$$

после несложных преобразований получаем равенство

$$
-\Delta\left(\psi-u_{1}\right)+q(x)\left(\psi-u_{1}\right)=0
$$

Неравенство $(27)$ и условия $(16),(18)$ и (19) дают неравенство $q(x)>0$ почти всюду на множестве $\bar{D}$. Далее, условие $(20)$ и совпадение на боковой границе цилиндра $Q$ функций $u(x, t)$ и $\mu(x, t)$ дают совпадение функций $\psi(x)$ и $u_{1}(x)$ на $Г$. Это совпадение, неравенство $q(x)>0$ и теорема единственности решений первой краевой задачи для эллиптических уравнений дают совпадение функций $\psi(x)$ и $u_{1}(x)$ почти всюду в области $D$.

Все сказанное означает, что функция $u(x, t)$, построенная с помошью решения краевой задачи (21)-(23), и функция $q(x)$, построенная по формуле $(29)$, дадут требуемое решение обратной задачи (11)-(14).

Теорема полностью доказана.

Приведем простейший пример, показывающий, что множество исходных данных обратной задачи (11)-(14), для которых вьполняются все условия теоремы 4 , непусто.

Пусть в обратной задаче (11)-(14) область $D$ лежит в некотором шаре $\{x:|x| \leqslant R\}$ пространства $\mathbb{R}^{n}, f(x, t)$ и $u_{0}(x)$ - тождественно нулевые соответственно в $\bar{Q}$ и $\bar{D}$ функции, $u_{1}(x)$ есть функция

$$
u_{1}(x)=k_{0}\left(|x|^{2}+1\right)^{\lambda}, \quad k_{0}>0, \quad \lambda \geqslant 1 .
$$

Далее, пусть $\bar{\mu}(t)$ и $\alpha(t)$ суть гладкие функции такие, что выполняется

$$
\bar{\mu}(0)=0, \quad \mu_{0}=\int_{0}^{T} \bar{\mu}(t) \alpha(t) d t>0 .
$$


Определим функцию $\mu(x, t)$ :

$$
\mu(x, t)=\frac{1}{\mu_{0}} \bar{\mu}(t) u_{1}(x) .
$$

Обозначим

$$
\mu_{1}=\max _{[0, T]}|\bar{\mu}(t)|
$$

Очевидны соотношения

$$
a(x)=\Delta u_{1} \geqslant m_{0}=2 \lambda n, \quad N_{0} \leqslant \frac{\mu_{1} k_{0}\left(R^{2}+1\right)^{\lambda}}{\mu_{0}} .
$$

В качестве числа $m_{1}$ возьмем число $\lambda n$. Имеет место неравенство

$$
N_{1} \leqslant \frac{a_{0} k_{0} \mu_{1}\left(R^{2}+1\right)^{2 \lambda}}{\lambda \mu_{0} n} .
$$

Очевидно теперь, что первое условие (19) будет заведомо выполняться при больших $n$. При выполнении первого условия (19) имеет место неравенство

$$
\frac{N_{2}}{1-N_{1}} \leqslant \frac{a_{0} k_{0}^{2} \mu_{1}^{2}\left(R^{2}+1\right)^{3 \lambda}}{\mu_{0}\left[\lambda \mu_{0} n-a_{0} k_{0} \mu_{1}\left(R^{2}+1\right)^{2 \lambda}\right]} .
$$

Вновь очевидно, что второе условие (19) также будет выполняться при больших $n$.

Возьмем теперь конкретные функции $\bar{\mu}(t)$ и $\alpha(t)$ :

$$
\bar{\mu}(t)=t, \quad \alpha(t)=t-t_{0}, \quad 0<t_{0}<\frac{2}{3} T .
$$

Имеем

$$
\mu_{1}=T, \quad a_{0}=2 T-t_{0}, \quad \mu_{0}=\frac{2 T^{3}-3 t_{0} T^{2}}{6} .
$$

Числа $m_{0}$ и $m_{1}$ зафиксируем как и ранее: $m_{0}=2 \lambda n, m_{1}=\lambda n$. Имеют место неравенства

$$
\begin{gathered}
N_{0} \leqslant \frac{6 k_{0} T\left(R^{2}+1\right)^{\lambda}}{2 T^{3}-3 t_{0} T^{2}}, \quad N_{1} \leqslant \frac{6 k_{0} T\left(2 T-t_{0}\right)\left(R^{2}+1\right)^{2 \lambda}}{\lambda n\left(2 T^{3}-3 t_{0} T^{2}\right)} \\
\frac{N_{2}}{1-N_{1}} \leqslant \frac{36 k_{0}^{2} T^{2}\left(2 T-t_{0}\right)\left(R^{2}+1\right)^{3 \lambda}}{\left(2 T^{3}-3 t_{0} T^{2}\right)\left[\lambda n\left(2 T^{3}-3 t_{0} T^{2}\right)-6 k_{0} T\left(2 T-t_{0}\right)\left(R^{2}+1\right)^{2 \lambda}\right]} .
\end{gathered}
$$

Очевидно теперь, что условия (19) будут выполняться, если число $T$ будет достаточно большим.

Сделаем два заключительных замечания.

ЗАмЕчАниЕ 2. В обратной задаче (11)-(14) условия положительности функций $u_{1}(x)$ и $a(x)$ можно заменить условиями отрицательности. Далее, в отличие от работы [11], мы не требуем положительности функции $\alpha(t)$ на всем отрезке [0,T]. Эта функция может обращаться в нуль, может принимать и отрицательные значения (в то же время вследствие условий (16) и (20) она не может быть тождественно нулевой).

ЗАмЕчАнИЕ 3. Условиегладкости на функцию $\alpha(t)$ в обратной задаче $(11)-(14)$ можно ослабить, потребовав лишь вьполнения условий $\alpha(t) \in L_{\infty}([0, T]), \alpha^{\prime}(t) \in L_{1}([0, T])$. 


\section{СПИСОК ЦИТИРОВАННОЙ ЛИТЕРАТУРЫ}

[1] Нахушев А. М. Уравнения математической физики. М.: Высшая школа, 1995.

[2] Дженалиев М. Т. К теории краевых задач для нагруженных дифференциальных уравнений. Алматы: Институт теоретической и прикладной математики, 1995.

[3] Cannon J. R., Yin H. M. On a class of nonlinear nonclassical parabolic problems // J. Differential Equations. 1989. V. 79. P. 266-288.

[4] Chadam J. M., Peirce A., Yin H. M. The blowup property of solutions to some diffusion equation with localized nonlinear reactions // J. Math. Anal. Appl. 1992. V. 169. № 2. P. 313-328.

[5] Kozhanov A. I. Inverse problem and "loaded" composite type equations // Нелинейные граничные задачи. 2000. Вып. 10. С. 109-116.

[6] Kozhanov A. I. Nonlinear inverse problems for elliptic equations // J. Inverse and Ill-Posed Problems. 2001. V. 9. № 4. P. 413-424.

[7] Шелухин В. В. Вариационный принцип в нелокальных по времени задачах для линейных эволюционных уравнений // Сиб. матем. ж. 1993. Т. 34. № 2. С. 191-207.

[8] Шелухин В. В. Нелокальные по времени задачи для уравнений гидродинамики и вариационные принципы. Дисс. ... д. ф.--м.н. Новосибирск, 1992.

[9] Ладыженская О. А., Солонников В. А., Уральцева Н. Н. Линейные и квазилинейные уравнения параболического типа. М.: Наука, 1967.

[10] Олейник О. А., Радкевич Е. В. Уравнения второго порядка с неотрицательной характеристической формой. Итоги науки. М.: ВИНИТИ, 1971.

[11] Прилепко А. И., Костин А. Б. Об обратных задачах определения коэффициентов в параболическом уравнении // Сиб. матем. ж. 1992. Т. 33. № 3. С. 146-155.

Институт математики им. С. Л. Соболева СО РАН, г. Новосибирск

Поступило

E-mail: kozhanov@math.nsc.ru

26.12 .2001 